\title{
EL TURISMO SOLIDARIO COMO INSTRUMENTO DE DESARROLLO: UN ESTUDIO DE CASO PARA ANALIZAR LAS PRINCIPALES MOTIVACIONES DE LOS TURISTAS SOLIDARIOS
}

\author{
Laura Fuentes-Moraleda ${ }^{1}$ \\ Ana Muñoz-Mazón ${ }^{2}$ \\ Sara Rodríguez-Izquierdo \\ Universidad Rey Juan Carlos. Madrid
}

\section{RESUMEN}

Este estudio se centra en el concepto y evolución del turismo solidario así como en las motivaciones del turista que lo realiza. Tras la revisión de la literatura y basado en un estudio cuantitativo de la cartera de clientes de una ONG española especializada en viajes solidarios, se concluye que la interactuación con otras culturas y el enriquecimiento personal son dos de las principales motivaciones de este tipo de viajero. Los resultados del estudio presentan algunas orientaciones para que las comunidades adapten su oferta actual hacia una mayor integración con el viajero.

Palabras clave: turismo solidario; motivación; solidaridad; ONGs; desarrollo local.

\section{Responsible tourism as an instrument for local development: a case study to analyze the main motivations for tourists}

\section{ABSTRACT}

This study is focused on the concept and evolution of responsible tourism and on the main motivations for tourists. Based on the literature review and on the quantitative

Recibido: 13 de enero de 2014

Devuelto para su revisión: 26 de junio de 2014

Aceptado: 19 de enero de 2015

1 Departamento de Economía de la Empresa. Facultad de Ciencias Jurídicas y Sociales. Universidad Rey Juan Carlos. Campus Fuenlabrada. C/ Camino del Molino s/n. 28943 Fuenlabrada. MADRID (España). E-mail: laura.fuentes@urjc.es

2 Departamento de Economía de la Empresa. Facultad de Ciencias Jurídicas y Sociales. Universidad Rey Juan Carlos. Paseo Artilleros s/n. 28032 Vicálvaro. MADRID (España). E-mail: ana.munoz@urjc.es 
study based on the clients data base of a Spanish NGO specialize in this kind of trips, it is concluded that the interaction between different cultures and the personal enrichment are two of the main motivations for this traveler. The results of this study present some guidelines for communities, in order to adapt their current supply to a greater integration with the traveler.

Keywords: responsible tourism; motivation; solidarity; NGOs; local development.

\section{INTRODUCCIÓN Y OBJETIVOS}

La actividad turística se caracteriza por su rápido y constante crecimiento en los últimos 50 años, consolidándose como un pilar clave en la economía de muchos países. Las consecuencias del crecimiento del turismo en otros sectores económicos no han sido ampliamente estudiadas, predominando una visión apriorística que asegura que el desarrollo turístico impulsa a los otros sectores de forma automática, aunque la realidad sea diferente en numerosas ocasiones (Gascón y Ojeda, 2014). En el escenario internacional, y ante una demanda cada vez más especializada en busca de experiencias únicas, han surgido nuevos modelos de oferta turística (Weiler y Hall, 1992; Robinson \& Novelli, 2005). Dentro de estos nuevos modelos se encuentran aquellos que fomentan la actividad turística como un instrumento de desarrollo local sobre las premisas de la sostenibilidad, entendida como la generación del menor impacto ambiental y sociocultural, junto a la generación de beneficios económicos en las comunidades receptoras. En este sentido, es importante entender los matices y las implicaciones de sostener que el turismo puede desempeñar -y de hecho desempeña- un papel en el desarrollo. El desarrollo es un proceso de transformación social que supone una acumulación e interacción de capital humano, institucional y físico (Muñoz - Mazón, Fuentes - Moraleda y Fayos - Solà, 2012).

Bajo este paradigma, han surgido iniciativas como son el turismo solidario, el turismo justo, el turismo ecológico, el ecoturismo, las brigadas solidarias y el voluntariado, entre otros. Todos ellos enmarcados dentro de un turismo responsable, respetuoso con el entorno, con la cultura y la sociedad donde se desarrollan, y donde el turista desempeña un rol diferente.

El turismo responsable se configura como un fenómeno que refuerza y valora las identidades y culturas locales, fortalece la participación de la comunidad e incita al entendimiento, conocimiento y mutuo aprendizaje entre turistas y residentes. La actividad turística así concebida se entiende como un intercambio solidario y se perfila como una estrategia válida para incorporar dentro de los procesos de desarrollo sostenible de los destinos.

Muchos de los trabajos sobre turismo responsable están centrados en el análisis de la motivación del turista (Broad, 2003; Brown \& Lehto, 2005; Campbell \& Smith, 2005; Galley \& Clifton, 2004; Söderman \& Sinead, 2008; Ureily, Reichel, \& Ron, 2003; Wearing, 2001, 2004). Otros se centran en la experiencia de participar en expediciones de conservación (Gray \& Campbell, 2007). Aún así, Fodness (1994) y Crompton (1979) ya sugierieron que era necesario conocer más sobre las motivaciones de los turistas con el fin de desarrollar los productos turísticos conforme a necesidades y deseos reales. 
Por ello, el presente trabajo tiene como objetivo explorar las motivaciones del turista cuando realiza viajes solidarios en el extranjero, tomando como base de análisis la información de los individuos que ya han realizado viajes responsables a través de una ONG española. Los resultados de este estudio pueden ser de utilidad a las comunidades que quieran desarrollar esta modalidad de turismo como alternativa a su desarrollo local, ya que contribuyen a la identificación de los factores motivacionales de los mercados potenciales, pudiendo, de este modo, facilitar la creación de una oferta más diversificada y enfocada por parte de las comunidades receptoras. Se pretende, además, profundizar en el conocimiento acerca de la motivación del turista solidario en España, y aportar más conocimiento en este ámbito, ya que la mayor parte de estudios en el área se ha realizado en países con una larga trayectoria en este ámbito (Australia, Gran Bretaña e Italia, entre otros).

\section{CONCEPTUALIZACIÓN DEL TURISMO SOLIDARIO}

El estudio del turismo solidario ha despertado gran interés entre los investigadores y académicos durante la última década, lo que se debe, en parte, al incremento de organizaciones que ofertan este tipo de viajes, propiciado por el aumento de la demanda de turistas que buscan experiencias auténticas que les reporten ciertos beneficios personales.

Holmes, Smith, Lockstone-Binney \& Baum (2010) conciben el turismo solidario como el realizado por los turistas cuya motivación principal es la práctica de la solidaridad de una manera altruista, viajando a otro destino en su propio país o en el extranjero. Según esta definición, el turismo solidario incluiría una amplia gama de actividades con un nexo común: la solidaridad del viajero. En esta misma línea, Wearing (2001) define el turismo solidario como el turismo que realizan las personas con una motivación especial (la ayuda o el alivio de la pobreza material de algunos grupos de la sociedad, la restauración de elementos locales de impacto negativo para la sociedad o la conservación del medio ambiente, entre otros). Esta tendencia de incorporar la solidaridad a la actividad turística viene respaldada por estudios como el de Herrero Amo (2003), que identifica la conexión entre turismo y solidaridad como una alternativa en la que la principal motivación del viajero es la solidaridad.

Se pueden identificar distintas modalidades o formas de turismo solidario, considerando que todas tratan un mismo fenómeno, y que todas ellas tienen a la solidaridad como la principal motivación de esta estancia (pudiendo entender por solidaridad la cooperación, la sensibilización, la ayuda, el trabajo voluntario hacia la comunidad, etc.) y se realiza durante periodos vacacionales de los participantes. Con base en las características anteriores, pueden diferenciarse diferentes modelos de viaje dentro del turismo solidario como los campos de trabajo, campos de solidaridad, voluntariado internacional, VolunTourism (a veces se utiliza como sinónimo de turismo voluntario y también en los casos en los que se dedica una pequeña parte del viaje al voluntariado), vacaciones voluntarias (la mayor parte del viaje o todo el viaje por completo se dedica al voluntariado), gap year voluntering que, según Jones (2004), se refiere a un descanso antes o después de los estudios universitarios que puede abarcar un periodo desde los 3 a 12 meses, donde los participantes combinan los viajes, el trabajo remunerado, el voluntariado y estudios). 
Cañada y Gascón (2003) conciben el turismo responsable como un movimiento social a favor de la sostenibilidad del turismo que debe ser impulsado tanto en el Norte como en el Sur, que se basa en la cooperación, el respeto a las culturas y en una economía justa y solidaria que favorezca el desarrollo sostenible. Según los autores, los elementos básicos que definen este tipo de turismo serían que: (i) trabaja para establecer modelos de desarrollo turístico sostenibles y específicos para cada zona de destino, (ii) denuncia los impactos negativos que el turismo conlleva o puede conllevar en las sociedades anfitrionas, así como la imagen distorsionada que los visitantes pueden hacerse de la realidad que han ido a conocer y (iii) valora y reclama la responsabilidad de turistas, tour-operadores, anfitriones e instituciones públicas a la hora de favorecer modelos turísticos sostenibles en el territorio.

El turismo responsable no es únicamente, por tanto, un tipo de turismo con potencialidad, sino más bien un movimiento social que pretende apoyar los modelos de desarrollo sostenibles en destinos y adaptarse a los entornos específicos para cada zona, teniendo en cuenta las variables sociales, económicas y medioambientales.

\section{ORIGEN Y EVOLUCIÓN DEL TURISMO SOLIDARIO}

La manifestación del turismo solidario, según Sancho (2007), se debe a la relación de solidaridad entre el turista y la población de acogida. El mismo principio de solidaridad se exprime en su mayor parte en el plano internacional, en un contexto de flujos turísticos norte/sur. Esta necesidad, según Herrero (2003) y Sancho (2007) surge por factores como: (i) la nueva necesidad social de «hacer algo por los demás», un nuevo «estilo» y filosofía de trabajo, (iii) una orientación a la sostenibilidad, solidaridad y justicia en la cooperación y ejecución de sus actividades y (iv) el auge de las ONG's, asociaciones humanitarias y de cooperación al desarrollo que sensibilizan y acercan a los ciudadanos a una realidad desconocida y hasta ahora poco accesible.

En España, el desarrollo y evolución del turismo solidario está íntimamente relacionado con el acercamiento de las organizaciones sociales al sector turístico. Gascón (2009) realiza una revisión de la evolución histórica de los viajes organizados por las organizaciones sociales en los últimos treinta años y según el autor, los viajes con motivo de solidaridad surgieron en España a principios de la década de los ochenta como movimientos de apoyo revolucionarios y de resistencia. Este tipo de viaje fue conocido como «brigadas de solidaridad» y el objetivo principal de las organizaciones que lo gestionan era estrechamente político y revolucionario. El activismo dejaba tiempo para el ocio y el deseo personal de conocer la cultura del país y en gran medida conocer los problemas sociales en los que se encuentra.

En la década de los noventa, según Gascón (2009), surge un nuevo movimiento alejándose del carácter político y revolucionario que se protagonizo durante los años ochenta que concebía el viaje como herramienta para la sensibilización que pretendía dar a conocer in situ la realidad de los países del Sur. Asimismo, surgían, como estrategia de las organizaciones sociales para obtener base social e ingresos propios, comunidades y grupos de interés del Sur que recibían a los turistas y gestionaban la actividad. Con este objetivo de carácter poco político o revolucionario, numerosas organizaciones 
y algunas agencias de viajes verán en este sector una importante acción complementaria para diversificar ingresos creando diferentes proyectos de cooperación. Actualmente la carga política de este tipo de turismo es mucho menor, pasando a un concepto más ético y solidario del viaje que supone un cambio en el perfil de la demanda, para la que la motivación principal es vivir puntualmente una experiencia alternativa que tienen un componente de solidaridad, pero sin plantearse necesariamente que tal experiencia pueda cambiar su modelo de vida o suponga un compromiso activo (Gómez y Sanahuja, 2001).

\section{OFERTA Y DEMANDA DEL TURISMO SOLIDARIO: CARACTERÍSTICAS BÁSICAS DEL MERCADO}

La actividad en torno al turismo solidario ha aumentado significativamente desde la década de los setenta (Wearing, 2004). Determinar el crecimiento y la tasa de mercado de este tipo de turismo es complejo, sin embargo, el aumento de las organizaciones turísticas responsables y actividades de voluntariado por parte de las ONG's demuestra que este sector turístico está en continuo crecimiento. De hecho, además de las acciones de la cooperación al desarrollo de dichas organizaciones, hoy en día pueden encontrarse una gran variedad de operadores turísticos y organizaciones que ofrecen algún tipo de viaje solidario en destinos ubicados fundamentalmente en entornos rurales.

La oferta de turismo solidario es altamente heterogénea, y esto hace que los productos no sean similares ni en su contenido, ni en su distribución espacial. Se trata de viajes no estandarizados y muy flexibles, cuya programación suele responder a las inquietudes y preferencias de los viajeros pero fundamentalmente a las necesidades de las comunidades receptoras. Normalmente, todos ellos siguen unos parámetros comunes de respeto hacia las poblaciones de destino. Entre las principales características destacan (i) formación previa al viaje, (ii) inclusión de visitas a proyectos de desarrollo y organizaciones solidarias, (iii) contratación de servicios turísticos locales, (iv) transparencia en los precios como principio básico, (v) número reducido de participantes por viaje y (vi) concentración de viajes a países del Sur.

Respecto de la demanda, lo que realmente motiva a un viajero a realizar este tipo de viajes es una cuestión compleja y desconcertante, sin embargo, como ya se ha comentado, muchos autores sugieren que la comprensión de estas motivaciones es de gran importancia para las entidades organizadoras. Las principales motivaciones del turista que realiza un viaje de turismo solidario están relacionadas con el altruismo (King \& Linch, 1998). Por otro lado, Stebbins (1996) considera turismo solidario es una actividad de ocio responsable y con fines de auto realización, ya que el individuo quiere obtener recompensas personales y sociales por la práctica de la actividad. Estas variables son muy valoradas también en los resultados de otras investigaciones (Broad, 2003; Caissie \& Halpenny, 2003; Campbell \& Smith, 2005; Wearing, 2001, 2004).

Las conclusiones de las investigaciones sobre motivación del turista solidario coinciden en que son diversas y contemplan desde el deseo de ayudar, hacer algo diferente a lo normal, conocer la autenticidad, adquirir experiencias, participar en los viajes y la aventura, aprender, la búsqueda de placer, el crecimiento personal, el intercambio con 
otras culturas y el desarrollo profesional del viajero (Broad, 2003; Brown \& Lehto, 2005; Caissie \& Halpenny, 2003; Campbell \& Smith, 2005; Wearing, 2001, 2004).

Wearing (2001) agrupa las categorías motivacionales de los participantes en vacaciones solidarias de acuerdo a los siguientes grupos: (i) altruismo; (ii) viaje; (iii) aventura; (iv) crecimiento personal; (v) intercambio cultural; (vi) aprendizaje y (vii) desarrollo profesional. Un enfoque más complejo y ampliamente adoptado es propuesto por «Volunteer Motivation Inventory» (VMI) de McEwin y Jacobsen-d'Arcy (2002), que proponen un conjunto de motivaciones generales, que a su vez incluyen elementos o atributos que son entendidos como los principales motivos de un viaje (en este caso de las actividades de voluntariado). Los autores plantearon 44 breves declaraciones a las que los voluntarios respondían con una puntuación sobre 5 puntos que van del «muy en desacuerdo» a «muy de acuerdo» en una escala de Likert.

El VMI significó una valiosa contribución para la comprensión de las complejas motivaciones que impulsan a los viajeros. Este estudio dio como resultados que los valores, basados en creencias profundamente arraigadas de la importancia de ayudar a otros (Clary, Snyder \& Ridge, 1992) fue la motivación más importante. Para el presente trabajo, se ha tomado como modelo el VIM combinado con la versión adaptada, introduciendo y modificando las declaraciones para este estudio en cuestión, y se ha hecho llegar a una muestra de turistas solidarios de la organización.

El conocimiento de las motivaciones de los turistas es un elemento de gran importancia para el fomento de este tipo de turismo, ya que en función del tipo de motivaciones de los turistas podrían segmentarse aquellos grupos que mayores posibilidades de apoyo local puedan tener para la comunidad. Finalmente, este aprendizaje serviría para conocer cómo puede cada segmento contribuir en el proceso de sensibilización tanto a los países del Norte, en el ámbito de la cooperación, como a los destinos del sur, a través de campañas de sensibilización y apoyo técnico a las comunidades.

\section{METODOLOGÍA}

La metodología del trabajo se divide en dos fases, una primera cualitativa y una segunda de carácter cuantitativo. La investigación se aborda desde un estudio del caso de una organización española sin ánimo de lucro, a través de una base de datos proporcionada por la misma y con información de todos los viajeros que han realizado turismo solidario con esta entidad.

La primera fase, la cualitativa, se llevó a cabo a través de la realización de diferentes entrevistas con las responsables de la organización mencionada, con el fin de obtener una información más completa tanto de los viajes como de su organización. Por otro lado, para la fase cuantitativa, y con el fin de obtener más información sobre el perfil del turista solidario y sus motivaciones, se diseñó un cuestionario de 27 preguntas estructuradas en tres bloques: (i) datos demográficos, (ii) proyecto y viaje realizado y (iii) evaluación de la experiencia. El cuestionario se distribuyó entre más de 600 individuos que habían realizado viajes con la ONG y en él se solicitaba a los encuestados la valoración de las motivaciones en una escala de 1 a 5 (siendo 1 -nada de acuerdo- y 5 -totalmente de acuerdo-) para la realización de los viajes solidarios. 


\section{Tabla 1}

FICHA TÉCNICA DEL ESTUDIO

\begin{tabular}{|c|c|c|c|}
\hline FASES & $\begin{array}{c}\text { TIPOS DE } \\
\text { INVESTIGACIÓN }\end{array}$ & INSTRUMENTOS & $\begin{array}{l}\text { Tratamiento de } \\
\text { la información }\end{array}$ \\
\hline FASE I & $\begin{array}{l}\text { INVESTIGACIÓN } \\
\text { CUALITATIVA }\end{array}$ & $\begin{array}{l}2 \text { Entrevistas en profundidad con } \\
\text { las responsables de la ONG. }\end{array}$ & $\begin{array}{l}\text { Análisis del } \\
\text { discurso }\end{array}$ \\
\hline \multirow{8}{*}{ FASE II } & $\begin{array}{l}\text { INVESTIGACIÓN } \\
\text { CUANTITATIVA }\end{array}$ & Encuesta a turistas solidarios & \multirow{8}{*}{$\begin{array}{l}\text { Programa } \\
\text { estadístico } \\
\text { SPSS } 15.0\end{array}$} \\
\hline & Población & 617 turistas solidarios de la ONG & \\
\hline & Ámbito geográfico & España & \\
\hline & Metodología & $\begin{array}{l}\text { Cuestionario administrado } \\
\text { electrónicamente. }\end{array}$ & \\
\hline & Tamaño de la muestra & $\begin{array}{l}\text { 250. Muestreo probabilístico } \\
\text { aleatorio simple }\end{array}$ & \\
\hline & Error del muestreo & $+/-4,87 \%$ & \\
\hline & Nivel de significación & $95,5 \% \mathrm{Z}=2(\mathrm{p}=\mathrm{q}=0,5)$ & \\
\hline & $\begin{array}{l}\text { Fecha del trabajo de } \\
\text { campo }\end{array}$ & $\begin{array}{l}\text { Desde el } 29 \text { de marzo al } 30 \text { de } \\
\text { abril de } 2012\end{array}$ & \\
\hline
\end{tabular}

Fuente: elaboración propia.

Las motivaciones incluidas en el cuestionario se basaron en las del VMI (Volunteer Motivation Inventory) de McEwin y Jacobsen-d'Arcy (2004). Tomando como base el VMI se adaptó un cuestionario con 29 items relacionados con los atributos asignados a las 11 motivaciones propuestas por McEwin and Jacobsen-D'Arcy para conocer las principales motivaciones de las personas que habían realizado turismo solidario ${ }^{1}$. Los motivos propuestos fueron los once detallados en la tabla 2. Cada motivo se compone de un grupo de atributos, que pueden observarse en la tabla (hay 4 motivaciones simples, esto es, con un solo atributo).

1 El inventario de motivaciones de voluntarios se desarrolló en 2002 por McEwin y Jacobsen-d'Arcy (2002). Se administró a un total de 2.444 voluntarios de 15 diferentes organizaciones, por lo que el citado estudio se configura como uno de los mayores estudios de motivaciones de voluntarios llevados a cabo en todo el mundo. 
Tabla 2

\section{MOTIVACIONES DEL TURISTA SOLIDARIO (MTS) CON ESCALA DE VALORACIÓN DE 1 A 5}

\begin{tabular}{|c|c|c|c|c|c|c|}
\hline Motivo & Atributo & 1 & 2 & 3 & 4 & 5 \\
\hline M1. Valores & $\begin{array}{l}\text { M1.A1.Siento que puedo hacer algo por ayudar a los demás } \\
\text { M1.A2.Mi familia ha estado siempre involucrada en aspectos } \\
\text { de solidaridad y ayuda } \\
\text { M1.A3.La solidaridad es una parte de mis valores como persona } \\
\text { M1.A4.No me gusta vivir ajeno a las situaciones de desventajas } \\
\text { que viven otras personas }\end{array}$ & & & & & \\
\hline $\begin{array}{l}\text { M2. Desarrollo } \\
\text { de la carrera } \\
\text { profesional }\end{array}$ & $\begin{array}{l}\text { M2.A1.Realizo este tipo de actividad porque creo que puede } \\
\text { abrirme oportunidades de trabajo } \\
\text { M2.A2.Usar mis habilidades y conocimientos para ayudar a los } \\
\text { demás } \\
\text { M2.A3.Voluntad de trabajar en equipo } \\
\text { M2.A4.Opciones de explorar una nueva vocación } \\
\text { M2.A5.Compañerismo }\end{array}$ & & & & & \\
\hline $\begin{array}{l}\text { M3. Crecimiento } \\
\text { personal }\end{array}$ & $\begin{array}{l}\text { M3.A1.Deseo de mejorar y entender la sociedad a los individuos } \\
\text { M3.A2.Una oportunidad que me enriquece como persona } \\
\text { M3.A3.Una experiencia vital que cambia la forma de ver el } \\
\text { mundo } \\
\text { M3.A4.Mejora la confianza en mí mismo }\end{array}$ & & & & & \\
\hline $\begin{array}{l}\text { M4. } \\
\text { Reconocimiento }\end{array}$ & $\begin{array}{l}\text { M4.A1.Ser reconocido y útil para la ONG es importante para } \\
\text { mí } \\
\text { M4.A2.Ser respetado por los demás voluntarios de la ONG es } \\
\text { importante para mí } \\
\text { M4.A3.Recibir un reconocimiento por mi labor y mi trabajo en } \\
\text { el proyecto es importante para mí }\end{array}$ & & & & & \\
\hline M5. Autoestima & $\begin{array}{l}\text { M5.A1.El deseo de sentirme mejor o sentirme necesitado por } \\
\text { otros } \\
\text { M5.A2.Una experiencia diferente que siempre he querido } \\
\text { realizar } \\
\text { M5.A3.Una experiencia que me hace sentir mejor persona } \\
\text { M5.A4.Una experiencia que me hace sentir ocupado }\end{array}$ & & & & & \\
\hline $\begin{array}{l}\text { M6. Interacción } \\
\text { social }\end{array}$ & $\begin{array}{l}\text { M6.A1.Poder interactuar con otras culturas de otros países } \\
\text { M6.A2.Convivir con gente } \\
\text { M6.A3.Descubrir lugares fascinantes }\end{array}$ & & & & & \\
\hline $\begin{array}{l}\text { M7. Reacción } \\
\text { frente a } \\
\text { situaciones de } \\
\text { injusticia }\end{array}$ & $\begin{array}{l}\text { M7.A1.Una experiencia que me ayuda a entender las } \\
\text { desigualdades sociales }\end{array}$ & & & & & \\
\hline M8. Reciprocidad & M8.A1.Interacción: dar y recibir & & & & & \\
\hline M9. Religión & M9.A1.Promovido por motivaciones religiosas & & & & & \\
\hline M10. Gobierno & $\begin{array}{l}\text { M10.A1.Me ayuda a entender los problemas sociales y políticos } \\
\text { de países en vías de desarrollo }\end{array}$ & & & & & \\
\hline $\begin{array}{l}\text { M11. } \\
\text { Comunidad }\end{array}$ & $\begin{array}{l}\text { M11.A1.Contribuir a las comunidades locales con mi aportación } \\
\text { económica a través de este tipo de actividad } \\
\text { M11.A2.Colaborar en tareas de ayuda que beneficien a la } \\
\text { comunidad local }\end{array}$ & & & & & \\
\hline
\end{tabular}

Fuente: elaboración propia. 


\section{ANÁLISIS DE LOS RESULTADOS}

A continuación se muestran los principales resultados del estudio cuantitativo llevado a cabo en esta investigación. Estos resultados se dividen en cuatro bloques: (i) perfil del encuestado, (ii) características del viaje, (iii) motivaciones y (iv) grado de satisfacción.

\subsection{Perfil socio-demográfico del turista solidario encuestado}

De la muestra objeto de estudio, un $80 \%$ fueron mujeres, con edades comprendidas en un $86 \%$ de los casos entre 26 y 40 años $(\bar{X}: 32,67$; d.t.: 5,06) y un $98,8 \%$ nacionalidad española. Se trata mayoritariamente de viajeros solteros $(65,2 \%)$ y con alto nivel de estudios (el 66,8\% de los encuestados posee estudios universitarios). De los encuestados con estudios universitarios, un 10,8\% habían estudiado magisterio, un 7,6\% derecho, el 6,4\% estudios de trabajo social y el $6 \%$ de psicología.

\subsection{Experiencia del turista y características del viaje}

El 83,6\% de los encuestados tan solo habían participado una vez en un viaje con motivación solidaria, mientras que el 16,4\% ya habían realizado anteriormente turismo con motivación solidaria. El periodo elegido por la mayoría de los turistas para realizar el viaje coincide con el periodo vacacional, de forma que el 35,2\% de los turistas han realizado su viaje en el mes de agosto seguido de julio con un 23,6\%. Le sigue el mes de noviembre representado por el $10,4 \%$ de los encuestados.

En 34,4\% de los casos, los viajes han tenido una duración media de 4 semanas, seguido del $25,2 \%$ en el que el viaje solidario ha tenido una duración de más de cuatro semanas. En cuanto a la actividad principal realizada en el destino, la mayoría de los encuestados (84\%) han respondido «actividades con niños» seguido de «excursiones para conocer la región» por un $19 \%$ de los encuestados. El comportamiento de hombres y mujeres respecto a las actividades en destino no presenta diferencias significativas.

\subsection{Motivaciones de los turistas solidarios (MTS)}

La valoración más alta fue para la variable «Poder interactuar con otras culturas de países diferentes» con una media de $4.668(\mathrm{dt}=.65)$. Además han tenido una evaluación relevante, "Una oportunidad que me enriquece como persona» con una media de 4,568 $(\mathrm{dt}=.72)$, $\mathrm{y}$ «Una experiencia diferente que siempre he querido realizar» con una media de $4,428(\mathrm{dt}=.84)$.

Los atributos menos valorados por los turistas solidarios han sido «Promovido por motivaciones religiosas» con una puntuación media de 1,308 $(\mathrm{dt}=.75)$, «Pienso que las habilidades y conocimientos que adquiera tras la experiencia pueden servirme para mi desarrollo profesional» con una media de 1,896 ( $\mathrm{dt}=1.06)$, y «Porque mi familia ha estado siempre involucrada en aspectos de solidaridad y ayuda» con una media de 2,152 $(\mathrm{dt}=1.15)$. 
Se realizó un análisis de las interrelaciones entre los factores motivaciones MTS, y para ello se procedió al análisis de correlaciones. De este modo, para un nivel de confianza del $1 \%$, las correlaciones son moderadas, siendo la más fuerte la que se produce entre los factores de «Interacción social» $\mathrm{y}$ «Crecimiento personal» (coef.Pearson=0,567). También se detectan correlaciones entre el factor «Reacción»y «Crecimiento personal» (coef.Pearson=0,556), «Gobierno»y «Reacción» (coef.Pearson=0,546) y por último entre «Autoestima»y «Reconocimiento» (coef.Pearson $=0,502)^{2}$.

Tabla 3

PRINCIPALES MOTIVACIONES DE LOS VIAJEROS

\begin{tabular}{lccccc}
\hline Recursos culturales & $\mathrm{N}$ & Mínimo & Máximo & Media & Desv. típ. \\
\hline $\begin{array}{l}\text { Poder interactuar con otras culturas de países } \\
\text { diferentes }\end{array}$ & 250 & 1 & 5 & 4,668 & 0,6507 \\
\hline $\begin{array}{l}\text { Una oportunidad que me enriquece como } \\
\text { persona }\end{array}$ & 250 & 1 & 5 & 4,568 & 0,7207 \\
\hline $\begin{array}{l}\text { Una experiencia diferente que siempre he } \\
\text { querido realizar }\end{array}$ & 250 & 1 & 5 & 4,428 & 0,8483 \\
\hline $\begin{array}{l}\text { Siento que puedo hacer algo por ayudar a los } \\
\text { demás }\end{array}$ & 250 & 1 & 5 & 4,344 & 0,8745 \\
\hline $\begin{array}{l}\text { Deseo de mejorar y entender la sociedad y a los } \\
\text { individuos }\end{array}$ & 250 & 1 & 5 & 4,328 & 0,8192 \\
\hline $\begin{array}{l}\text { Estar dispuesto a convivir con la gente } \\
\text { No me gusta vivir ajeno ante las situaciones } \\
\text { desfavorecidas que pasan otras personas }\end{array}$ & 250 & 1 & 5 & 4,256 & 0,9043 \\
\hline $\begin{array}{l}\text { Porque la solidaridad es parte de mis valores } \\
\text { como persona }\end{array}$ & 250 & 1 & 5 & 4,2 & 0,8917 \\
\hline $\begin{array}{l}\text { Usar mis habilidades y el conocimiento para } \\
\text { ayudar a los demás }\end{array}$ & 250 & 1 & 5 & 4,196 & 0,8629 \\
\hline $\begin{array}{l}\text { Una experiencia vital que cambia mi forma de } \\
\text { ver el mundo }\end{array}$ & 250 & 1 & 5 & 4,18 & 0,9671 \\
\hline $\begin{array}{l}\text { Una forma de descubrir lugares fascinantes } \\
\text { Entender los problemas sociales y políticos de } \\
\text { países en vía de desarrollo }\end{array}$ & 250 & 1 & 5 & 4,168 & 1,1103 \\
\hline $\begin{array}{l}\text { Colaborar en tareas de ayuda que beneficien a la } \\
\text { comunidad local }\end{array}$ & 250 & 1 & 5 & 4,1 & 0,9869 \\
\hline $\begin{array}{l}\text { Una experiencia que me ayuda a entender las } \\
\text { desigualdades sociales }\end{array}$ & 250 & 1 & 5 & 4,084 & 0,9637 \\
\hline \begin{tabular}{l} 
Voluntad de trabajar en equipo \\
\hline
\end{tabular} & 250 & 1 & 5 & 3,932 & 0,9936 \\
\hline
\end{tabular}

2 Para un nivel de significación del 5\% resultan significativas las correlaciones entre los factores de «Valor» $\mathrm{y}$ «Autoestima» (coef.Pearson=0,150), «Reconocimiento» $\mathrm{y}$ «Reacción» (coef.Pearson $=0,139)$ y entre «Comunidad» $\mathrm{y}$ «Autoestima» (coef.Pearson=0,153) pero resultan muy débiles. 


\begin{tabular}{lccccc}
\hline Interacción: dar y recibir & 250 & 1 & 5 & 3,872 & 1,0373 \\
\hline $\begin{array}{l}\text { Una experiencia que me hace sentir mejor } \\
\text { persona }\end{array}$ & 250 & 1 & 5 & 3,72 & 1,1829 \\
\hline $\begin{array}{l}\text { Compañerismo } \\
\text { Ser respetado por los demás voluntarios de la }\end{array}$ & 250 & 1 & 5 & 3,712 & 1,1036 \\
\hline $\begin{array}{l}\text { ONG es importante para mí } \\
\text { Mejorar la confianza en uno mismo }\end{array}$ & 250 & 1 & 5 & 3,28 & 1,3025 \\
\hline $\begin{array}{l}\text { Opciones de explorar una nueva vocación } \\
\text { Contribuir a las comunidades locales con mi } \\
\text { aportación económica a través de este tipo de } \\
\text { actividad }\end{array}$ & 250 & 1 & 5 & 3,168 & 1,2971 \\
\hline $\begin{array}{l}\text { El deseo de sentirse mejor o sentirse necesitado } \\
\text { por otros }\end{array}$ & 250 & 1 & 5 & 2,876 & 1,2756 \\
\hline $\begin{array}{l}\text { Ser reconocido y útil por la ONG es importante } \\
\text { para mí }\end{array}$ & 250 & 1 & 5 & 2,752 & 1,2493 \\
\hline $\begin{array}{l}\text { Ser reconocida mi labor y trabajo en el proyecto } \\
\text { es importante para mí }\end{array}$ & 250 & 1 & 5 & 2,58 & 1,1803 \\
\hline $\begin{array}{l}\text { Una experiencia que me hace mantenerme } \\
\text { ocupado }\end{array}$ & 250 & 1 & 5 & 2,188 & 1,2160 \\
\hline $\begin{array}{l}\text { Porque mi familia ha estado siempre } \\
\text { involucrada en aspectos de solidaridad y ayuda }\end{array}$ & 250 & 1 & 5 & 2,152 & 1,1551 \\
\hline $\begin{array}{l}\text { Pienso que las habilidades y conocimientos que } \\
\text { adquiera tras la experiencia pueden servirme } \\
\text { para mi desarrollo profesional }\end{array}$ & 250 & 1 & 5 & 1,896 & 1,0666 \\
\begin{tabular}{l} 
Promovido por motivaciones religiosas \\
\hline
\end{tabular} & 250 & 1 & 5 & 1,308 & 0,7533 \\
\hline
\end{tabular}

Fuente: elaboración propia.

\subsection{Grado de satisfacción y fidelización del turista con los viajes solidarios}

La valoración de la satisfacción por parte de los encuestados fue elevada, 4,05 puntos sobre 5 (d.t.:0,964). Esto se refleja en el hecho de que el 97, 6\% de los encuestados repetirían la experiencia de realizar un viaje con motivación solidaria, mientras que un 2,4\% que responde que no. Además, el 96,8\% recomendaría el viaje solidario a otras personas.

\section{Tabla 4}

GRADO DE SATISFACCIÓN CON EL PROYECTO ELEGIDO

\begin{tabular}{cccccc}
\hline Satisfacción proyecto & N & Mínimo & Máximo & Media & Desv. típ. \\
\hline & 250 & 1 & 5 & 4,05 &, 964 \\
\hline
\end{tabular}

Fuente: elaboración propia. 
Con el objeto de conocer si tras la experiencia de turismo solidario, continuaron con actividades relacionadas, se incluyó una pregunta para conocer cuál había sido su último destino vacacional. El 36,6\% de los turistas encuestados viajó a Europa, seguido del 22,1\% que viajó a Sudamérica. En cuanto al tipo de turismo que realizó, el 42,8\% optó por un turismo cultural, y un 13,2\% visita a familiares y amigos. El 55,2\% de los encuestados lo realizó por cuenta propia, el 30,4\% organizó el viaje a través de Internet.

Asimismo, se procedió a preguntar al turista si había continuado haciendo alguna actividad solidaria. Más de la mitad de los encuestados (58\%) había realizado actividades solidarias después del viaje frente a un $42 \%$ que no lo hizo. Además se preguntó si después del viaje se había continuado realizando alguna actividad solidaria (no turística). El $17 \%$ de los encuestados afirma que participa como voluntario en su sentido más general, el 15,2\% colabora con distintas ONG y el 13,3\% no realiza ningún tipo de actividad solidaria. Le siguen la colaboración económica con un 9,1\%, la sensibilización con un 9,1\% y un proyecto educativo con un $7,3 \%$.

\section{Tabla 5 \\ CONTINGENCIA ENTRE EXPECTATIVAS \\ DEL VIAJE Y GRADO DE SATISFACCIÓN}

\begin{tabular}{|c|c|c|c|c|c|c|c|}
\hline \multicolumn{8}{|c|}{ Grado de satisfacción } \\
\hline & $\begin{array}{r}\text { Tota } \\
\text { des }\end{array}$ & ente en & $\begin{array}{l}\text { Bastante en } \\
\text { desacuerdo }\end{array}$ & $\begin{array}{c}\text { Ni de } \\
\text { acuerdo ni en } \\
\text { desacuerdo }\end{array}$ & $\begin{array}{l}\text { Bastante de } \\
\text { acuerdo }\end{array}$ & $\begin{array}{l}\text { Totalmente } \\
\text { de acuerdo }\end{array}$ & Totales \\
\hline \multirow{3}{*}{$\begin{array}{c}\text { ¿Se ha cumplido } \\
\text { sus expectativas } \\
\text { del viaje? }\end{array}$} & No & $18,5 \%$ & $25,9 \%$ & $51,9 \%$ & $3,7 \%$ &, $0 \%$ & $100,0 \%$ \\
\hline & Sí &, $0 \%$ & $1,8 \%$ & $14,8 \%$ & $40,4 \%$ & $43,0 \%$ & $100,0 \%$ \\
\hline & Totales & $2,0 \%$ & $4,4 \%$ & $18,8 \%$ & $36,4 \%$ & $38,4 \%$ & $100,0 \%$ \\
\hline
\end{tabular}

Fuente: elaboración propia.

\section{CONCLUSIONES}

Para finalizar este trabajo es conveniente destacar sus hechos más significativos, de forma que permitan concluir los principales hallazgos e implicaciones del mismo. La primera conclusión del trabajo está relacionada con el concepto de turismo solidario. El turismo solidario se puede definir como el realizado por los viajeros cuya motivación principal es la práctica de la solidaridad de una manera altruista, viajando a otro destino en su propio país o al extranjero. Se pueden identificar distintas modalidades de turismo solidario (campos de trabajo, campos de solidaridad, voluntariado internacional, entre otras), pero todas ellas tienen a la solidaridad como la principal motivación de la estancia (pudiendo entender por solidaridad la cooperación, la sensibilización, la ayuda, el trabajo voluntario hacia la comunidad, etc) y se realiza durante periodos vacacionales de los participantes.

La segunda conclusión del trabajo está relacionada con las motivaciones de los viajeros para realizar turismo solidario. Los resultados del trabajo reflejan que el poder 
interactuar con otras culturas de países diferentes y el enriquecimiento personal son las principales motivaciones de las personas que realizan viajes motivados por la solidaridad. Estos resultados además coinciden con los de trabajos previos. Esta necesidad de sumergirse en la cultura local y en la comunidad también fue resaltada en las investigaciones de Brown y Lehto (2005) y Lo y Candy Y.S. Lee (2011). Dicha necesidad se complementa con la de poder hacer algo por los demás, que también fue concluido por el estudio de Clary, Snyder \& Ridge (1992).

Según lo anterior, aquellas comunidades o poblaciones que contemplen este tipo de actividad como un motor de desarrollo local, sería interesante que desarrollen actividades que permitan al turista poder interactuar con su cultura, tradiciones y forma de vida ya que esto es una oportunidad que les enriquece como personas. Vivir una experiencia diferente respecto a la que pueden vivir en cualquier otro tipo de viaje «convencional» que puedan realizar, unido al sentimiento de poder hacer algo por ayudar a los demás, son aspectos claramente diferenciales que definen claramente a este tipo de viajeros y marcan la decisión sobre su viaje.

En este sentido, las comunidades han de valorar aquellas costumbres, formas de vida, labores cotidianas y otras manifestaciones de su folclore como los elementos clave alrededor que los cuales hacer girar las experiencias del turista solidario que, además de estar vinculado con un proyecto concreto (trabajo en escuelas, centros de formación u otros), desea tener una experiencia integral con la comunidad receptora. A modo de ejemplo, la integración en las labores de elaboración de alimentos típicos, en sus ritos y costumbres, en los trabajos de recogida de productos agrícolas, de trabajo con los animales, o cualquier otra actividad cotidiana que lleve a cabo la comunidad debe ser valorada por la misma y ofertada (en la medida de lo posible) a los turistas que deseen una vivencia auténtica y real.

La tercera conclusión está relacionada con las principales actividades llevadas a cabo durante la estancia en el destino. La mayoría de los encuestados (84\%) realizaron actividades con niños, debido al carácter de los proyectos, seguido de excursiones para conocer la región (19\% de los encuestados), lo que refleja las necesidades de los viajeros relacionadas con conocer los principales atractivos de la zona independientemente del tipo de proyecto concreto en el que se esté trabajando. Esto puede provocar que, dentro de la propia comunidad, nazcan iniciativas de emprendedores locales que brinden este tipo de servicios a los turistas. De esta forma, si el servicio es prestado por los propios miembros de la comunidad, la comunidad se verá beneficiada directamente. La prestación de estos servicios requiere la formación específica de jóvenes en profesiones como guías turísticos o en la hostelería, que les brinde los conocimientos y la capacidad de conocer más sobre sus propias costumbres y lugares más atractivos, de su gastronomía y la historia vinculada a la misma... y poder transmitirlo a los turistas. Hoy día existen diversas iniciativas tanto en España como en otros países que involucran a los turistas en el cultivo de diferentes alimentos, plantas medicinales, en la elaboración de alimentos, en la producción de sus artesanías, en el cuidado de sus animales, y esto les reporta a los turistas y a la comunidad beneficios en todos los sentidos (económicos, sociales y ambientales).

Las comunidades y poblaciones que trabajan con turistas solidarios, además de alojar a los turistas y vinculares con proyectos de campo concretos, deberían realizar, de manera previa, una labor de identificación de atractivos locales (rutas y senderos, cuevas, avista- 
miento de especies animales locales, restos arqueológicos...) y formarse y capacitarse para poder prestar un servicio de calidad a los turistas que están conviviendo en la comunidad. Estas actividades pueden generar nuevos puestos de trabajo y permiten diversificar la economía local, además de producir interacciones con los turistas, lo que redundaría en un mayor crecimiento personal.

Finalmente, cabe destacar la elevada satisfacción por parte de los encuestados $(4,05$ puntos sobre 5), lo que se refleja en el hecho de que el 97, 6\% de los encuestados repetirían la experiencia, y que a su vez explicaría el hecho de que el 96,8\% recomendaría el viaje solidario a otras personas. El alto grado de intención de repetición además coincide con los resultados de otros estudios, donde se muestra que la percepción del valor de la visita a un destino o participar en un tipo especifico de viajes influye fuertemente en la intención futura de los viajeros de volver al mismo destino o volver a participar en una experiencia de viaje similar (Petrick, 2004).

\section{REFERENCIAS BIBLIOGRÁFICAS}

BROAD, S. (2003): «Living the Thai life e a case study of volunteer tourism at the Gibbon Rehabilitation Project », Tourism Recreation Research, n 28(3), pp. 63-72.

BROAD, S. y JENKINS, J. (2009): Gibbons in their midst? Conservation volunteers' motivations at the Gibbon rehabilitation project, Phuket, Thailand. En K. D. Lyons, \& $\mathrm{S}$. Wearing (Eds.), Journeys of discovery in volunteer tourism international case study perspectives. Wallingford: CABI.

BROWN, S. y LEHTo, X. (2005): «Travelling with a purpose: understanding the motives and benefits of volunteer vacationers», Current Issues in Tourism, ${ }^{\circ}$ 8(6), pp. 479-496.

BRUYERE, B. y RAPPE, S. (2007): «Identifying the motivations of environmental volunteers», Journal of Environmental Planning and Management, $\mathrm{n}^{\circ}$ 50(4), pp. 503-516.

CAISSIE, L.T. y HALPENNY, E.A. (2003): «Volunteering for nature: motivations for participating in a biodiversity conservation volunteer program», World Leisure Journal, $\mathrm{n}^{\mathrm{o}} 45(2)$, pp. 38-50.

CAMPBELL, L.M., y SMITH, C. (2005): «What makes them pay? Values of volunteer tourists working for sea turtle conservation», Environmental Management, n 38(1), pp. 84-98.

CAÑADA, E. y GASCÓN, J. (2003). Turismo Responsable. Guía Temática de Oneworld.

CLARY, E.G. SNYDER, M. y RIDGE R. (1992): «Volunteers Motivations; A Functional Strategy for the Recruitment, Placement and Retention of Volunteers», Nonprofit Management and Leadership, Vol. 2 (4), pp. 335-51.

CLARY, E.G. y SNYDER, M. (1999): «The motivations to volunteer: theoretical and practical considerations». Current Directions in Psychological Science, $\mathrm{n}^{\circ}$ 8(5), pp 156-159.

CROMPTON, J.L. (1979): «Motivations for pleasure vacation». Annals of Tourism Research, 6(1), 408-424.

DANN, G.M.S. (1981): «Tourism motivation: an appraisal», Annals of Tourism Research, $\mathrm{n}^{\mathrm{o}} 8(2), \mathrm{pp} .187-219$.

DRIVER, B.L. y KNOPF, R.C. (1977): «Personality, outdoor recreation and expected consequences». Environmental behavior. Pp. 169-193. 
DRIVER, B.L, TINSLEY, H.E. y MANFREDO, M.J. (1991): The paragraphs about leisure and recreation experience preference scales: Two inventories designed to assess the breadth of the perceived psychological benefits of leisure. Capítulo de Libro «Benefits of Leisure» State College, Pa.: Venture Pub.

FODENESS, D. (1994): «Measuring Tourist Motivation». Annals of Tourism Research, $\mathrm{n}^{\mathrm{o}} 21(3), \mathrm{pp} .555-81$.

GALLEY, G. y CLIFTON, J. (2004): «The motivational and demographic characteristics of research ecotourists: Operation Wallacea volunteers in Southeast Sulawesi, Indonesia», Journal of Ecotourism, no 3(1), pp. 69-82.

GASCÓN, J. (2009): El turismo en la cooperación internacional: de las brigadas internacionales al turismo solidario. Relaciones Norte-Sur. ISBN: 9788498880717.

GASCÓN, J. y OJEDA, D. (2014): «Turistas y campesinado: el turismo como vector de cambio de las economías campesinas en la era de la globalización». PASOS. Revista de Turismo y Patrimonio Cultural. №12. Colección Thesis. ISBN (impresa): 978-8488429-25-4.

GÓMEZ M. y SANAHUJA, J.A (2001): La cooperación al desarrollo en un mundo en cambio. Reflexiones desde nuevos ámbitos de intervención. Madrid, CIDEAL.

GRAY, N.J. y CAMPBELL, L.M. (2007): «A decommodified experience? Exploring aesthetic, economic and ethical values for volunteer ecotourism in Costa Rica», Journal of Sustainable Tourism, $\mathrm{n}^{\circ}$ 15(5), pp. 463-482.

HERRERO, M.D. (2003): Turismo Solidario. Marco de Aproximación. Diploma Estudios Avanzados en Turismo. Madrid, España.

HOLMES, K., SMITH, K.A., LOCKSTONE-BINNEY, L. y BAUM, T. (2010): «Developing the dimensions of tourism volunteering», Leisure Sciences, $\mathrm{n}^{\circ} 32(3)$, pp. 255-269.

HORTON-SMITH, D. (1981): «Altruism, Volunteers and Volunteerism», Nonprofit and Voluntary Sector Quarterly n ${ }^{\circ}$ 10(1), pp. 21-36.

ISO-AHOLA, S.E. (1989): Understanding leisure and recreation: Mapping the past, charting the future. En Motivation for Leisure. Jackson, Edgar L.; Burton, Thomas L.. State College, PA: Venture Publishing Inc.

KING, K. N. y LYNCH, C.V. (1998): «The motivations of volunteers in The Nature Conservancy--Ohio Chapter, a non-profit environmental organization», The Journal of Volunteer Administration, $\mathrm{n}^{\circ}$ 16(2), pp. 5-11.

LO, A. S. y LEE, C.Y.S. (2011): «Motivations and perceived value of volunteer tourists from Hong Kong». Tourism Management, $\mathrm{n}^{\mathrm{o}}$ 32(2), pp 326-334.

MCEWIN, M. y JACOBSEN-D'ARCY, L. (1992): Developing a scale to understand and assess the underlying motivational drives of volunteers in Western Australia, Perth. Lotterywest and CLAN inc.

MUÑOZ-MAZÓN, A., FUENTES-MORALEDA, L. y FAYOS-SOLÀ E. (2012) «Turismo como instrumento de desarrollo: Una visión alternativa desde factores humanos, sociales e institucionales». Pasos. Revista de Turismo y Patrimonio Cultural, vol. 10, $\mathrm{n}^{\circ} 5$, pp. 437-469.

NOVELLI, M. (2005): Niche Tourism. Contemporary Issues, Trends and Cases. Elsevier Butterworth-Heinemann. Burlington. MA. USA 
PEARCE, P.L., y CALTABIANO, M. (1983): «Inferring Travel Motivations from Travelers' Experiences», Journal of Travel Research, Vol. 22 (2), pp. 16-20.

PEARCE, P.L. y LEE, U. (2005). «Developing the travel career approach to tourist motivation», Journal of Travel Research $\mathrm{n}^{\circ} 43$ (3), pp. 226-237.

PETRICK, J.F. (2004): «The roles of quality, value and satisfaction in predicting cruise passengers' behavioral intentions», Journal of Travel Research, $\mathrm{n}^{\circ}$ 42(4), pp. 397-407.

RIECKEN, G., BABAKUS, E. y YAVAS, U. (1994): «Facing resource attraction challenges in the non-profit sector: a behavioristic approach to fundraising and volunteer recruitment», Journal of Professional Services Marketing, $\mathrm{n}^{\circ}$ 11(1), pp. 45-70.

ROBINSON, M. y NOVELLI, M. (2005): Niche Tourism: An introduction En: Novelli, M., ed. Niche Tourism: contemporary issues, trends and cases. Butterworth-Heinemann Ltd., Oxford, UK, pp. 1-14. ISBN 0-7506-6133-X

SANCHO, A. (2007): Turismo y Desarrollo. Ponencia en las Jornadas Internacionales de Turismo Justo: Un reto para el desarrollo. Universidad de Valencia.

SÖDERMAN, N. y SNEAD, S. (2008): Opening the gap: The motivation of gap year travellers to volunteer in Latin America. En K. D. Lyons \& S. Wearing (Eds.), Journeys of discovery in volunteer tourism: International case study perspectives (pp. 118-129). Wallingford, UK:CABI.

STEBBINS, R.A (1996): «Cultural Tourism as Serious Leisure», Annals of Tourism Research v23 n4: pp. 948-950.

UREILY, N., REICHEL, A. y RON, A. (2003): «Volunteering in tourism, additional thinking» Tourism Recreation Research, 28(3), pp. 57-62.

WEARING, S. (2000): «Refiguring Self and Identity through Volunteer Tourism», Loisir et Societe 23, pp. 389-419.

WEARING, S. (2001): Volunteer Tourism: Experiences That Make a Difference. CABI Publishing, Wallingford.

WEARING, S. (2002): Re-centering the Self in Volunteer Tourism. En: Dann,G. (ed.) The Tourist as a Metaphor of the Social World. CABI Publishing, Wallingford, pp. 237-262. WEARING, S. (2004): Examining best practice in volunteer tourism. En R. A. Stebbins, WEILER, B. y HALL C.M. (1992): Special interest tourism: in search of an alternative. En B. Weiler y C. M. Hall (Eds.). Special interest tourism (pp. 194-204). London. Belhaven Press. 